\title{
Orientational Ordering of a Dimer Liquid Crystal by High-Resolution Solid-State ${ }^{13}$ C NMR Spectroscopy
}

\author{
Renato Norio Shimizu, Hiromichi Kurosu, Isao Ando, ${ }^{\dagger}$ Akihiro ABE, ${ }^{*}$ \\ Hidemine FurUYa, and Shigeki KUROKI** \\ Department of Polymer Chemistry, Tokyo Institute of Technology, \\ 2-12-1 Ookayama, Meguro-ku, Tokyo 152, Japan. \\ * Department of Industrial Chemistry, Tokyo Institute of Polytechnics, \\ 1583 Iiyama, Atugi-shi 243-02, Japan \\ ** Advanced Polymer Laboratory, Japan High Polymer Center, \\ 2-1-6 Sengen, Tsukuba 305, Japan
}

(Received December 9, 1996)

\begin{abstract}
The ordering of a dimer liquid crystal, $\alpha, \omega$-bis $\left[\left(4,4^{\prime}\right.\right.$-cyanobiphenylyl)oxy $]$ decane (CBA-10), was studied by variable-temperature high-resolution solid-state ${ }^{13} \mathrm{C}$ NMR. A large transitional change of the ${ }^{13} \mathrm{C}$ chemical shift was observed on going from the isotropic to the nematic phase due to uniaxial alignment of the molecule. The principal values of the ${ }^{13} \mathrm{C}$ chemical shift tensor have been determined by the Herzfeld-Berger method for CBA-10 itself. The molecular axis is assumed to lie parallel to the line connecting the centers of both mesogenic cores, and the evaluation of the order parameters along the molecular axis, $\left(S_{z z}\right)$, mesogene axis $\left(S_{z z}^{\text {ring }}\right)$ and $\mathrm{C}_{\gamma}-\mathrm{C}_{\varepsilon}$ axis $\left(S_{z z}^{\mathrm{C}_{\gamma}-\mathrm{C}_{\varepsilon}}\right)$ was attempted here, using the obtained isotropic ${ }^{13} \mathrm{C}$ chemical shift and principal values of the ${ }^{13} \mathrm{C}$ chemical shift tensor, and taking into account the conformational behavior of the flexible spacer in the frame of rotational isomeric state (RIS) approach. The order parameter of the molecule $S_{z z}$ increased from 0.79 to 0.89 with decrease of $17.5^{\circ} \mathrm{C}$ in temperature from nearby the nematic-isotropic transition point. $S_{z z}^{\text {ring increased }}$ from 0.74 to 0.80 with decrease of temperature whereas $S_{z z}^{C_{\gamma}-C_{\varepsilon}}$ and the conformation of the spacer was essentially unaltered in the same temperature range.

KEY WORDS High-Resolution Solid-State ${ }^{13} \mathrm{C}$ NMR / ${ }^{13} \mathrm{C}$ Chemical Shift Tensor / Conformation / Rotational Isomeric State / Order Parameter / Dimer Liquid Crystal /
\end{abstract}

Low molecular weight monomer liquid crystals are composed of rod-like molecules which form an uniaxially oriented mesophase. The chemical shift anisotropy of the ${ }^{13} \mathrm{C}$ nucleus has proved to be a powerful tool for probing the orientational ordering at the molecular level of such compounds ${ }^{1,2}$ and for a side-chain liquid-crystal polymer. ${ }^{3}$ Though the principal values of the chemical shift tensor needed for the analysis are in some cases assumed to be the same for analogous compounds, this may not be true ${ }^{2.4}$ and may lead to error in constructing models of microscopic configurations from the observed data.

In the case of dimer liquid crystals consisting of two mesogenic moieties linked by a flexible alkyl chain, as the model of polymer liquid crystals, the molecules align as a whole in the nematic phase, the conformation of the spacer being quite stable in a large temperature range. ${ }^{5}$

Our goal in this work is to prepare a ${ }^{13} \mathrm{C}$-labeled dimer liquid crystal, $\alpha, \omega$-bis $\left[\left(4,4^{\prime}\right.\right.$-cyanobiphenylyl)oxy $]$ decane (CBA-10) and to analyze its ordering behavior through the observation of the ${ }^{13} \mathrm{C}$ chemical shift anisotropy, determining the principal values of the ${ }^{13} \mathrm{C}$ chemical shift tensor for CBA-10 itself. This is the first attempt with a dimer liquid crystal in which the conformation of the spacer must be considered in the analysis of ordering being different from simple monomer liquid crystals. Spacer conformation is considered in the frame of the rotational isomeric state (RIS) approach.

\footnotetext{
$\dagger$ To whom all correspondence should be addressed.
}

\section{EXPERIMENTAL}

\section{Materials}

The preparation of $\alpha, \omega$-bis [(4,4'-cyanobiphenylyl)oxy]decane and of $\alpha, \omega$-bis[(4,4'-cyanobiphenylyl)oxy]decane- $\delta-{ }^{13} \mathrm{C}_{2}$ was accomplished by reacting 4-hydroxy$4^{4}$-cyanobiphenyl with 1,10-dibromodecane and ${ }^{13} \mathrm{C}$ labeled 1,10-dibromodecane-4,7- ${ }^{13} \mathrm{C}_{2}$ respectively as reported elsewhere. ${ }^{5}$

The 1,10-dibromodecane-4,7- ${ }^{13} \mathrm{C}_{2}$ was prepared from butanedioic acid-1, $4-{ }^{13} \mathrm{C}_{2} 99$ atom $\%{ }^{13} \mathrm{C}$ purchased from Nippon Sanso Co., Ltd. First, 1,4-dibromobutane-1,4${ }^{13} \mathrm{C}_{2}$ was synthesized by standard methods reducing the butanedioic acid-1,4- ${ }^{13} \mathrm{C}_{2}$ with excess lithium aluminium hydride to 1,4-butanediol-1,4- ${ }^{13} \mathrm{C}_{2}$ and reacting the obtained diol with excess phosphorous tribromide. The obtained dibromide was reacted with 1-butenylmagnesium bromide producing 1,11-dodecadiene-5, $8-{ }^{13} \mathrm{C}_{2}$. The catalyst dilithium tetrachlorocuprate was used and the reaction was conducted under the conditions reported by Zimmermann. ${ }^{6}$ The resulting diene was submitted to ozonolisis followed by a reduction with sodium boron hydride producing 1,10 -decanediol-4,7- ${ }^{13} \mathrm{C}_{2}$ which was turned into 1,10 -dibromodecane- $4,7-{ }^{13} \mathrm{C}_{2}$ by reaction with excess phosphorous tribromide.

\section{Measurements}

High-resolution solid-state ${ }^{13} \mathrm{C}$ NMR spectra were measured on a JEOL-GSX270 NMR spectrometer (67.8 $\mathrm{MHz}$ ) equipped with a variable temperature accessory. Measurements above room temperature were carried out on a CP/MAS probe from Doty Scientific, Inc. The temperature of the sample was determined from calibra- 
tion $^{7}$ using thermolabels from Nichiyugiken Kogyo Co., Ltd. In the present study the sample was not spun. In Figure 1 the temperature inside the probe $\left(T_{\text {real }}\right)$ is plotted against the value indicated in the console $\left(T_{\text {ind }}\right)$. The relation between the two is

$$
T_{\text {real }}=1.04 T_{\text {ind }}-6.7\left({ }^{\circ} \mathrm{C}\right)
$$

In experiments using MAS the relation reported by Akieda $^{7}$ was used.

A Bruker DSX300 $(75.5 \mathrm{MHz})$ spectrometer with low MAS rate $(350 \mathrm{kHz})$ was used as well. The ${ }^{13} \mathrm{C}$ chemical shifts were referred to an external adamantane reference (29.5 ppm relative to TMS). The cross polarization contact time was set to $2 \mathrm{~ms}$ and the repetition times were $5 \mathrm{~s}$ and $10 \mathrm{~s}$ respectively for CP/MAS and PST (pulse saturation transfer) experiments. In the PST method a single pulse excitation with presaturation of the proton spins is used. ${ }^{8}$ The intensity of the signals due to the mobile carbons is thus enhanced. The rf strength varied in between $1.3-1.5 \mathrm{mT}$ for ${ }^{1} \mathrm{H}$ for both $\mathrm{CP}$ and decoupling processes and $5.1-6.0 \mathrm{mT}$ for ${ }^{13} \mathrm{C}$. Also, the ${ }^{13} \mathrm{C} \mathrm{CP} / \mathrm{MAS}$ spectra with total suppression of sidebands (TOSS) and total suppression of protonated carbons (MASDL) were measured so as to assign the spinning sidebands (SSB). In the TOSS method the spinning sidebands are suppressed, ${ }^{9}$ and in the MASDL method dipolar dephasing is used so as to suppress the signals due to the protonated carbons. ${ }^{10}$

\section{RESULTS AND DISCUSSION}

The resonance frequency of a nuclear spin $I$ is a function of the surrounding electrons. In the case of ${ }^{13} \mathrm{C}$ nucleus in the samples studied here the electron distribution is not spherically symmetric and the electronic shielding is anisotropic. Therefore, the so called shielding constant is a second-rank tensor and can be represented pictorially as an ellipsoid. The position of a particular resonance line in a spectrum depends on the angles between the static magnetic field direction and the ellipsoid semi-axes (the principal axes of the tensor) and on the lengths of these axes (the principal values of the tensor).

For aromatic carbons the most shielded direction is normal to the aromatic ring and the least shielded one is in the plane of the ring. For the methylene carbons, the least shielded direction is parallel to the interprotons vector. The intermediately shielded direction lies along the bisector of $\mathrm{H}-\mathrm{C}-\mathrm{H}$ angle (see Figure 6). The mag-

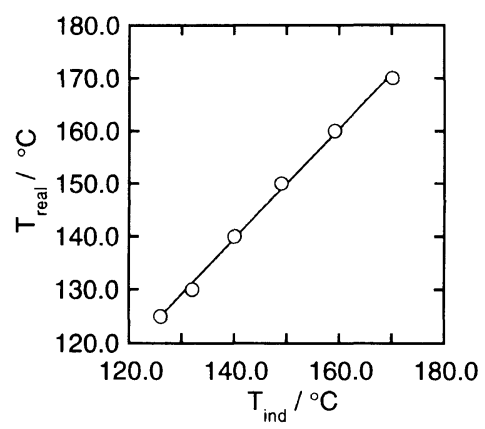

Figure 1. Correlation between temperature inside the NMR probe, $T_{\text {real }}$, and that indicated in the console of the instrument. netic susceptibility, $\Delta \chi$, of CBA-10 is positive ${ }^{11}$ and therefore in the nematic phase, the alignment of the molecules is with the long axis (director) parallel ${ }^{12}$ to the strong magnetic field $\vec{B}_{0}$. Hence on going from the isotropic to the nematic phase, a downfield shift is expected for the peaks due to the aromatic carbons in relation to their positions in the isotropic phase due to the alignment of the molecules. For the aliphatic carbons, on the contrary, an upfield shift is expected.

In Figure $3{ }^{13} \mathrm{C}$ NMR spectra of CBA-10 taken at different temperatures are presented. The assignments of the resonance lines were based on results previously reported $^{13}$ (see Figure 2 for the numbering of the carbons). The signal of $\mathrm{C}_{\delta}$ was assigned by measuring the $99 \%{ }^{13} \mathrm{C}$ enriched sample at $\mathrm{C}_{\delta}$. The signal due to $\mathrm{C}_{\alpha}$ is seen near $70 \mathrm{ppm}$. The signals for the other carbons are not assigned. However they are not used in this study. As can be seen here the peaks for the aromatic carbons show a downfield, and for the aliphatic ones an upfield shift indicating the alignment of the molecule.

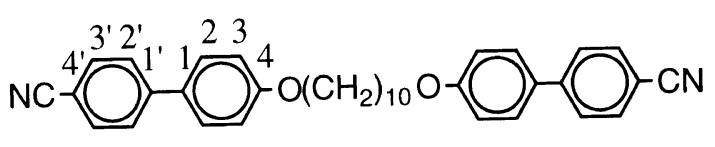

CBA-10

Figure 2. Numbering of carbons in CBA-10.

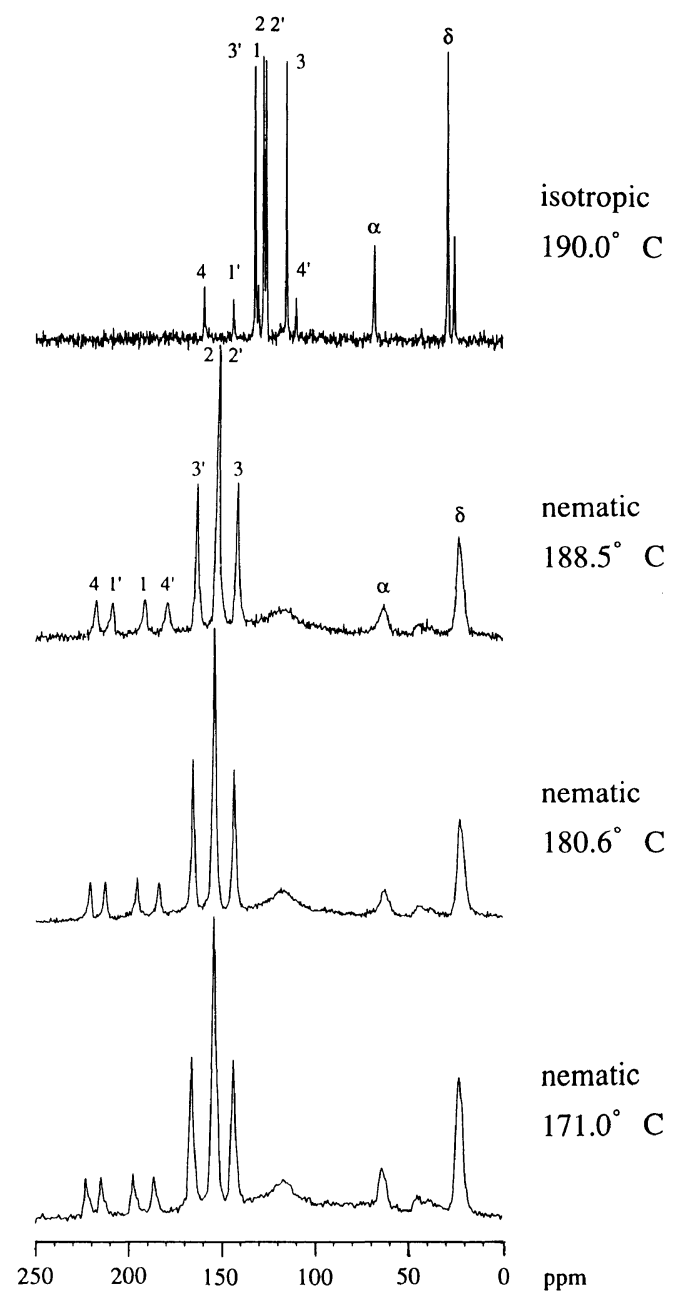

Figure 3. High-resolution solid-state ${ }^{13} \mathrm{C}$ NMR spectra of CBA-10 observed at different temperatures without MAS. 


\section{Determination of the Principal Values of the ${ }^{13} \mathrm{C}$ Chemical Shift Tensor}

As pointed out previously, the assumption that the principal values $\left(\delta_{11}, \delta_{22}\right.$, and $\left.\delta_{33}\right)$ of the chemical shift tensor are the same among unlike compounds may introduce large error in the calculation of the order parameter. Hence, the use of accurate values of $\delta_{11}$, $\delta_{22}$, and $\delta_{33}$ is of key importance.

We determined the principal values directly for CBA10 itself by the Herzfeld and Berger method, ${ }^{14}$ i.e., highresolution solid-state ${ }^{13} \mathrm{C}$ NMR measurements of CBA10 powder sample have been carried out, rotating the sample at the magic angle at low spinning rates so as to generate spinning sidebands. In Figure $4 a$, the sample was rotated at $1800 \mathrm{~Hz}$ and the asterisks indicate the SSBs of $\mathrm{C}_{1}$, carbon signal. The assignment of the signals was based in previous results. ${ }^{13}$ In the case of the aliphatic carbon, CBA-10 powder sample with both $\delta$ positions of the spacer labeled with ${ }^{13} \mathrm{C}$ has been used and so as to obtain the sideband pattern shown in Figure $5 a$ the sample was spun at $350 \mathrm{~Hz}$ at the magic angle.
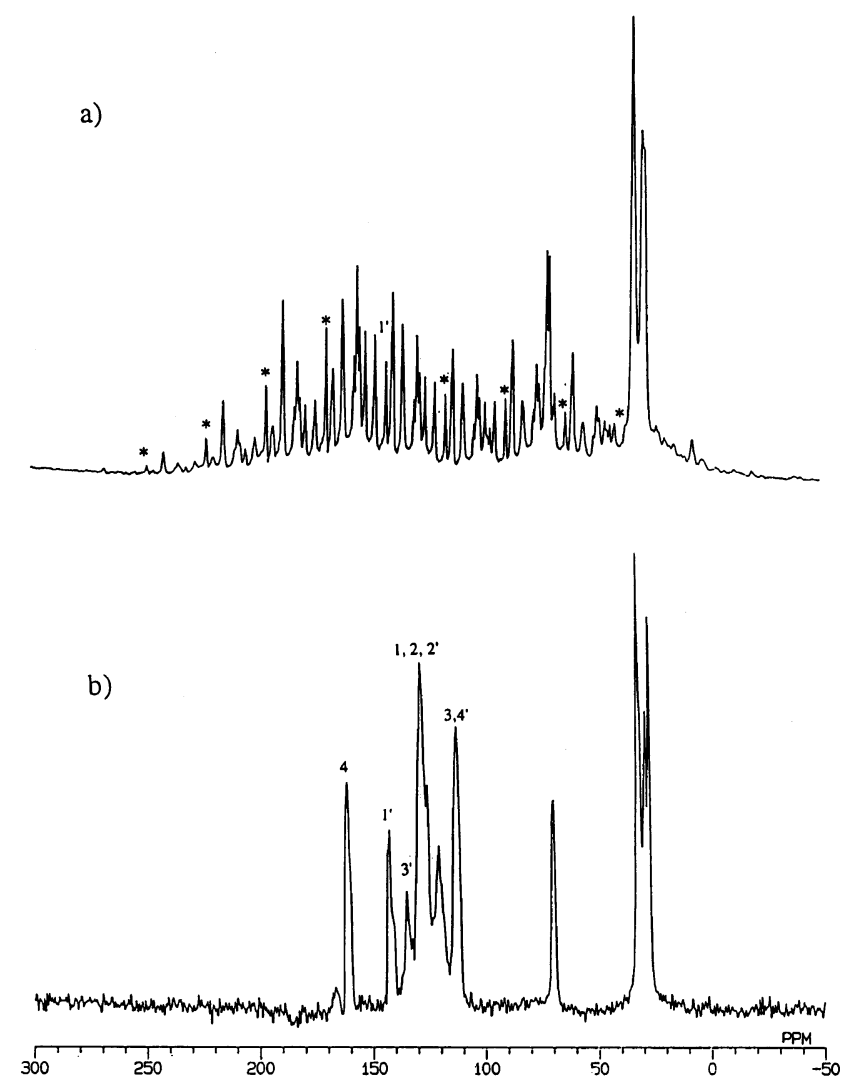

Figure 4. High-resolution solid-state ${ }^{13} \mathrm{C}$ NMR spectra of CBA-10 powder sample: a) CP/MAS spectrum taken at MAS rate of $1800 \mathrm{~Hz}$. The SSBs of the $\mathrm{C}_{1}$, carbon are indicated with an asterisk; b) TOSS spectrum.

Table I. Principal values of the ${ }^{13} \mathrm{C}$ chemical shift tensor (ppm) for CBA-10 and interior methylene groups in $n-\mathrm{C}_{20} \mathrm{H}_{42}$, relative to TMS

\begin{tabular}{crrr}
\hline Carbon & \multicolumn{1}{c}{$\delta_{11}$} & \multicolumn{1}{c}{$\delta_{22}$} & $\delta_{33}$ \\
\hline $\mathrm{C}_{1}{ }^{\prime}$ & 228.7 & 162.3 & 36.1 \\
$\mathrm{C}_{\delta}$ & 45.5 & 34.9 & 17.4 \\
$n-\mathrm{C}_{20} \mathrm{H}_{42}{ }^{\mathrm{a}}$ & 50.2 & 38.2 & 17.2 \\
\hline
\end{tabular}

${ }^{a}$ From ref 15.
The simulation of these sideband patterns permitted us to determine the principal values of the chemical shift tensor of $\mathrm{C}_{1}$, and $\mathrm{C}_{\delta}$ carbons with high accuracy. The values relative to TMS are shown in Table I. For comparison, the values reported ${ }^{15}$ for the interior methylene groups of $n$-eicosane are presented together. They are quite different from those determined for $\mathrm{C}_{\delta}$ carbon in this study.

\section{The Calculation of the Order Parameters}

Assuming that the ordering of the whole molecule can be described by a single ordering matrix, the chemical shifts of each carbon in the liquid crystalline $\delta_{\mathrm{LC}}$ and isotropic $\delta_{\text {iso }}$ states are related to the order parameters of the molecule by the following equation,

$$
\delta_{\mathrm{LC}}=\delta_{\text {iso }}+\frac{2}{3} \sum_{\alpha=x}^{z} \sum_{\beta=x}^{z} S_{\alpha \beta} \delta_{\alpha \beta}
$$

which is obtained by the coordinate system transformation from the molecular axis system to the laboratory frame and by considering motional averaging of the orientation of the molecules. Here, $x, y$, and $z$ are the axes attached to the molecule and $S_{\alpha \beta}$ s are the elements of the Saupe ordering matrix.

Equation 2 can be expanded as in eq 3; here assuming that $\left(S_{x x}-S_{y y}\right)$ is negligible compared to $S_{z z}$ and molecular motion is fast around the long molecular axis, the third and fourth terms may be neglected and so eq 3 is simplified and rewritten as eq 4 ,

$$
\begin{aligned}
\delta_{\mathrm{LC}}= & \delta_{\text {iso }}+\frac{2}{3} S_{z z}\left\{\delta_{z z}-\frac{1}{2}\left(\delta_{x x}+\delta_{y y}\right)\right\} \\
& +\frac{1}{3}\left(S_{x x}-S_{y y}\right)\left(\delta_{x x}-\delta_{y y}\right) \\
& +\frac{2}{3} \sum_{\substack{\alpha=x \\
\alpha \neq \beta}}^{z} \sum_{\substack{\beta \neq x \\
\alpha \neq \beta}}^{z} S_{\alpha \beta} \delta_{\alpha \beta} \\
S_{z z} & =\frac{(3 / 2)\left(\delta_{\mathrm{LC}}-\delta_{\text {iso }}\right)}{\left\{\delta_{z z}-(1 / 2)\left(\delta_{x x}+\delta_{y y}\right)\right\}}
\end{aligned}
$$

where $\delta_{x x}, \delta_{y y}$, and $\delta_{z z}$ are the chemical shift tensor elements in the molecular axis system and calculated from the principal values $\left(\delta_{11}, \delta_{22}\right.$, and $\left.\delta_{33}\right)$ through system transformation from the principal axis to molecular axis system.

Following our previous analysis scheme, ${ }^{5}$ the long molecular axis $(z)$ of CBA-10 is assumed to be parallel to the line connecting the midpoints of both mesogenes as indicated in Figure 6 . In the case of a dimer liquid crystal the relative positions of the molecular axis and principal axis system depend on the conformation of the flexible spacer. This has been considered in the frame of rotational isomeric state (RIS) approach, i.e., statistical weights $\sigma_{i}, i=1$ to 6 have been defined for the gauche conformation of each bond (see Figure 6). The geometrical parameters for the description of the spacer have been reported elsewhere ${ }^{5}$, and the following values have been used for the conformational energy: $E_{\sigma_{1}}=1.4 \mathrm{kcal} \mathrm{mol}^{-1}$, $E_{\sigma_{2}}=0 \mathrm{kcal} \mathrm{mol}^{-1}$ and $E_{\sigma_{i}}=0.5 \mathrm{kcal} \mathrm{mol}^{-1}, i=3$ to 6 . Second-order interactions are assumed to be completely suppressed under the nematic constraints and therefore, conformers presenting sequences such as $g^{+} g^{-}$or $g^{-} g^{+}$ 
are not considered in the calculations.

Initially, all the possible conformers have been generated in the calculations; then the order parameter of the

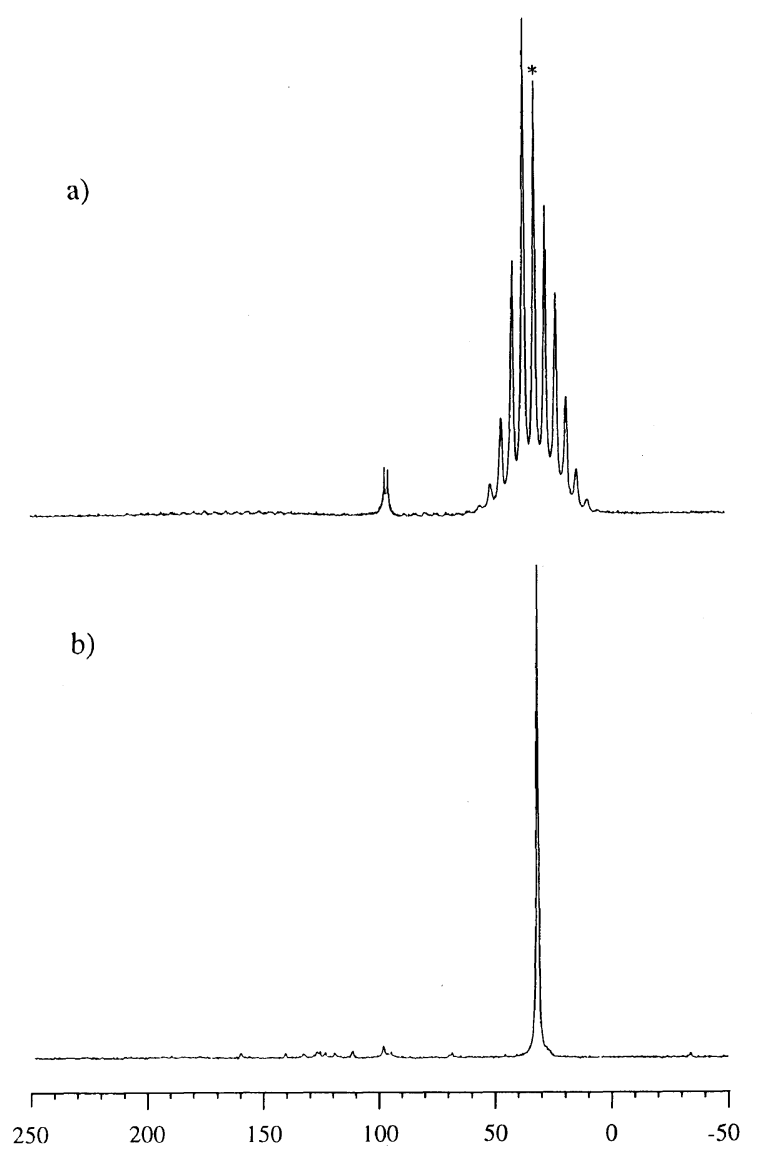

Figure 5. $\mathrm{CP} / \mathrm{MAS}$ spectra of ${ }^{13} \mathrm{C}$ labeled $\mathrm{CBA}-10$ powder sample at both $\delta$ positions of the flexible spacer: a) with MAS rate of $350 \mathrm{~Hz}$. Main peak is indicated with an asterisk; b) with MAS rate of $5000 \mathrm{~Hz}$.

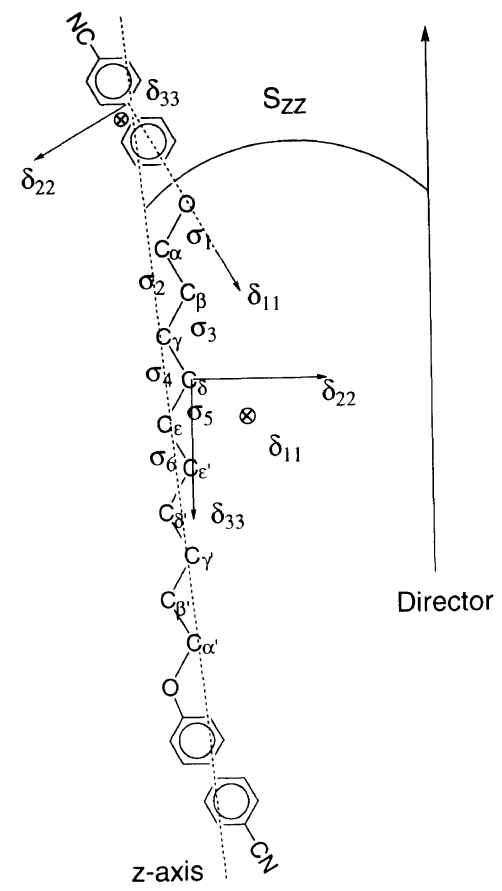

Figure 6. Definition of molecular axis and statistical weights $\sigma_{i}$ assigned to the gauche state of each bond and the direction of the principal axes of the chemical shift tensor for an aromatic and an aliphatic carbons. molecular axis of each conformer was calculated by eq 4 , using the chemical shift information about both the $\mathrm{C}_{1}$, and $\mathrm{C}_{\delta}$ carbons. Conformers presenting a negative value or a value greater than 1 for the calculated order parameter were discarded in this step and conformers with the order parameters calculated from the chemical shifts of $\mathrm{C}_{1}$, and $\mathrm{C}_{\delta}$ carbons differing from each other by a value greater than an error $\varepsilon$ ( $\varepsilon$ was set in 0.01 ) in the absolute terms were excluded as well. Finally, the order parameter was calculated as a statistical average of the conformers thus selected. The orderings of the mesogenes and of the $\mathrm{C}_{\gamma}-\mathrm{C}_{\varepsilon}$ axis were calculated by the following expression and compared with the values calculated directly.

$$
S_{z z}^{i}=S_{z z}\left(3\left\langle\cos ^{2} \alpha\right\rangle-1\right) / 2
$$

where $S_{z z}^{i}$ is the order parameter of the moiety in question, $\alpha$ is the angle between the molecular axis and the moiety axis and the angular brackets indicate statistical average.

The error bars in Figures 7 to 10 are based on the consideration of the uncertainties in the chemical shift values. All the calculations were done in two different molecular axis systems having the $z$ axis in common so as to test the assumption that the system exhibits axial symmetry. The difference in the results from both the systems was found to be within the range indicated by the above mentioned error bars, thus confirming the validity of our assumption.

In Figure 7 the order parameter of the molecular axis is plotted against temperature. It can be seen that the order parameter increases with decreasing temperature. For the mesogenes also a decrease in temperature results in an increase in the ordering as shown in Figure 8. Here,

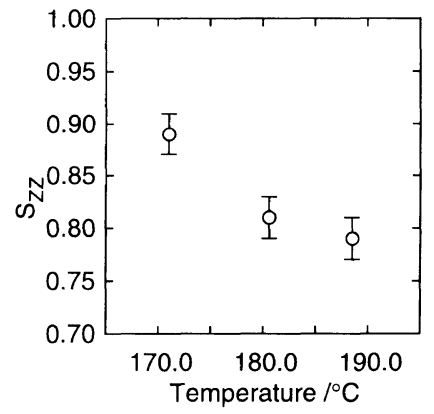

Figure 7. Dependence of the order parameter of the molecular axis on temperature. The error bars are based on uncertainty in the values of chemical shifts.

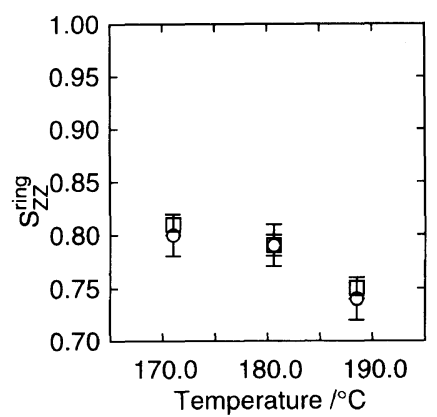

Figure 8. Dependence of the order parameter of the mesogenic moiety on temperature. Circles indicate the values calculated from the order parameter of the molecular axis. Squares indicate values calculated directly (see the text). 


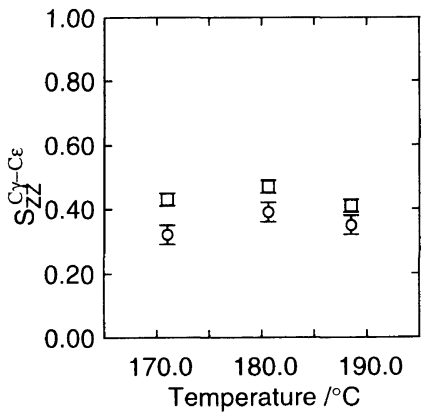

Figure 9. Dependence of the order parameter of the $C_{\gamma}-C_{\varepsilon}$ axis on temperature. Circles indicate values calculated from the order parameter of the molecular axis. Squares indicate values calculated directly (see the text).

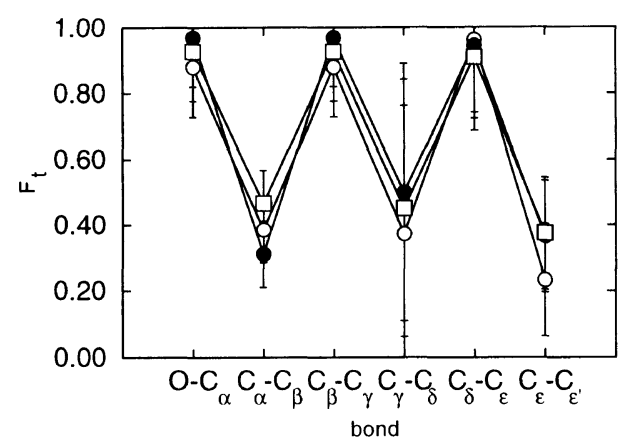

Figure 10. The fraction of trans conformation $F_{1}$ for each bond, at different temperatures. Open circles indicate values for $171.0^{\circ} \mathrm{C}$; filled circles indicate values for $180.6^{\circ} \mathrm{C}$ and open squares, values for $188.5^{\circ} \mathrm{C}$.

the open circles indicate the values calculated using eq 5 and open squares are values calculated directly using eq 4 being the para axis of the biphenyl moiety the $z$ axis. The agreement between both values is within experimental error.

In both cases (molecular axis and mesogene) the order parameter estimated in this study is greater than the value reported previously ${ }^{5}: c a .0 .2$ higher in the case of the molecular axis and ca. 0.3 higher for the mesogenic moiety over the temperature range studied. It shoud be noted that to consider the value of the biaxiality $S_{x x}-S_{y y}$ in the calculations implies in an increase in $S_{z z}$ value, although small, in both the cases. In the present analysis, it is not possible to evaluate the value of $S_{x x}-S_{y y}$ by the chemical shift information alone.

In the case of the $\mathrm{C}_{\gamma}-\mathrm{C}_{\varepsilon}$ axis, however, the order parameter stays essentially constant with temperature and is lower than the values obtained for the mesogenes as shown in Figure 9. The values directly calculated and those derived from the order parameter of the molecular axis do not agree within experimental uncertainity.

The temperature dependence of the trans fraction is very small (see Figure 10). The error bars in $\mathrm{C}_{\gamma}-\mathrm{C}_{\delta}$, and $\mathrm{C}_{\varepsilon}-\mathrm{C}_{\varepsilon^{\prime}}$ bonds are greater compared to the other bonds because they have small effect on the alignment of the mesogenes. This conformation is compatible, within experimental error, with the results reported previously ${ }^{5}$ except for $\mathrm{C}_{\beta}-\mathrm{C}_{\gamma}$ and $\mathrm{C}_{\delta}-\mathrm{C}_{\varepsilon}$ bonds which have great influence on the alignmnet of the both mesogenic moieties.

Finally, analysis of the influence of the geometrical parameters was made. Increase in the torsional angles of the bonds in the spacer resulted in almost no changes in the conformation of the spacer. The same is true for the values of $S_{z z}, S_{z z}^{\text {ring }}$, and $S_{z z}^{\mathrm{C}_{\gamma}-\mathrm{C}_{\varepsilon}}$. In the case of increase in the bond angles, there was hardly any change in $S_{z z}$, $S_{z z}^{\text {ring }}$ and in the conformation. However, $S_{z z}^{\mathrm{C}_{\gamma}-\mathrm{C}_{\varepsilon}}$ reached the value directly calculated from eq 4 . This may explain somewhat the lack of agreement for $S_{z z}^{\mathrm{C}_{\gamma}-\mathrm{C}_{\varepsilon}}$ mentioned above.

\section{CONCLUSION}

A method for estimating the order parameter of a dimer liquid crystal by analysis of the ${ }^{13} \mathrm{C}$ chemical shift anisotropy is proposed. Analysis of $\alpha, \omega$-bis $\left[\left(4,4^{\prime}-\right.\right.$ cyanobiphenylyl)oxy]decane clearly showed the orderings of the mesogenes and of the molecule itself to increase with decreasing temperature whereas that of the $\mathrm{C}_{\gamma}-\mathrm{C}_{\varepsilon}$ axis to remain essentially constant. The conformation of the spacer remains almost unaltered with decreasing temperature in agreement with previous results. $^{5}$

Acknowledgments. The authors are indebted to Professor Takeshi Nakai and Dr. Katsuhiko Tomooka of Department of Chemical Engineering of Tokyo Institute of Technology for invaluable discussion and assistance in the synthesis of the $\alpha, \omega$-bis [(4,4'-cyanobiphenylyl)oxy $]$ decane- $\delta-{ }^{13} \mathrm{C}_{2}$. The authors are grateful to Professor Bernard C. Gerstein of Ames Laboratory, Minerals and Mining Resources Research Institute for helpful advice and suggestions.

\section{REFERENCES}

1. A. Pines and J. J. Chang, J. Am. Chem. Soc., 96, 5590 (1974); A. Pines and J. J. Chang, Phys. Rev. A, 10, 946 (1974); J. W. Emsley, "Measurement of Orientational Ordering by NMR" in "Nuclear Magnetic Resonance of Liquid Crystals," J. W. Emsley, Ed., Reidel, Dordrecht, 1985, p 379.

2. C. -D. Poon and B. M. Fung, Liq. Crystals, 5, 1159 (1989).

3. H. Oulyadi, F. Lauprêtre, L. Monnerie, M. Mauzac, H. Richard, and H. Gasparoux, Macromolecules, 23, 1965 (1990).

4. B. M. Fung and C. F. Kong, J. Am. Chem. Soc., 106, 6193 (1984); W. S. Veeman, Prog. NMR Spectrosc., 16, 193 (1984).

5. A. Abe, R. N. Shimizu, and H. Furuya, "Phase Diagram and Molecular Ordering of Dimer Liquid Crystals Dissolved in a Simple Nematic Solvent," in "Ordering in Macromolecular Systems," A. Teramoto, M. Kobayashi, T. Norisuye, Ed., Springer-Verlag, Berlin, Heidelberg, 1994, p. 139; A. Abe, H. Furuya, R. N. Shimizu, and S. Y. Nam, Macromolecules, 28, 96 (1995).

6. H. Zimmermann, Liq. Crystals, 4, 591 (1989).

7. T. Akieda, H. Mimura, S. Kuroki, H. Kurosu, and I. Ando, Macromolecules, 25, 5794 (1992).

8. T. Fujito, K. Deguchi, M. Ohuchi, M. Imanari, and M. J. Albright, in "Proceedings of the 20th NMR Meeting," Tokyo, 1981, p 68.

9. W. T. Dixon, J. Chem. Phys., 77, 1800 (1982).

10. L. B. Alemany, D. M. Grant, T. D. Alger, and R. J. Pugmire, J. Am. Chem. Soc., 105, 6697 (1983); S. J. Opella and M. H. Frey, J. Am. Chem. Soc., 101, 5854 (1979).

11. H. Furuya, T. Dries, K. Fuhrmann, A. Abe, M. Ballauff, and E. W. Fischer, Macromolecules, 23, 4122 (1990).

12. J. Courtieu, D. W. Alderman, D. M. Grant, and J. P. Bayles, J. Chem. Phys., 77, 723 (1982).

13. C. -D. Poon, J. Afzal, M. Gangoda, and B. M. Fung, Magn. Res. Chem., 24, 1014 (1986); J. S. Lewis, E. Tomchuk, and E. Bock, Mol. Cryst. Liq. Cryst., 198, 191 (1991).

14. J. Herzfeld and A. E. Berger, J. Chem. Phys., 73, 6021 (1980).

15. D. L. VanderHart, J. Chem. Phys., 64, 830 (1976). 